\title{
Performance of Inverse Problem Regularization Methods for Driver Location during Atrial Fibrillation
}

\author{
Carlos Figuera ${ }^{1}$, Victor Suárez-Gutiérrez ${ }^{1}$, Óscar Barquero-Pérez ${ }^{1}$, Rebeca Goya-Esteban ${ }^{1}$, \\ Miguel Rodrigo ${ }^{2}$, Ismael Hernández ${ }^{1,2}$, Felipe Atienza ${ }^{3}$, María S. Guillem ${ }^{2}$, Andreu M. Climent ${ }^{3}$, \\ Felipe Alonso-Atienza ${ }^{1}$. \\ ${ }^{1}$ Dept. of Signal Theory and Communications, Universidad Rey Juan Carlos, Madrid, Spain \\ ${ }^{2}$ ITACA, Universitat Politècnica de València, València, Spain \\ ${ }^{3}$ Instituto de Investigación Sanitaria, Hospital Gregorio Marañón, Madrid, Spain
}

\begin{abstract}
Locating the atrial fibrillation $(A F)$ sources is a relevant and not fully analyzed problem.

We propose a procedure to benchmark methods for driver location in $A F$ and compared three representative techniques: zero-order Tikhonov, Greensite and Bayes (maximum a posteriori). These methods were used to estimate the epicardial potentials, in turn used to locate the driver, using a realistic computer model for atria and torso with two simulated $A F$ propagation patterns.

The assessment is based on the spatial mass function of the driver location (SMF), i.e. the probability of the driver being at each point of the atria. Being the driver region $(D R)$ the points with $S M F>0$, we defined three metrics: (i) weighted under-estimation indicator, which is the weighted percentage of the true DR that is not detected out of the entire true DR; (ii) the weighted over-estimation indicator, which is the percentage of the misjudged DR out of the entire estimated DR; and (iii) the correlation coeffcient between real and estimated SMFs.

Results show that the these metrics are easy to compute and provide representative information about the location accuracy. Among the compared algorithms, Bayes method provided the best performance in both AF patterns.

Remarkably, even for the most complex pattern, for which epicardial potentials estimation was inaccurate, the three methods approximately located the activity driver.
\end{abstract}

\section{Introduction}

Atrial fibrillation (AF) is the most common arrhythmia in clinical practice and it is associated with an increased risk of embolism, cardiac failure and mortality [1]. Recently, some works have focused on techniques for locating AF drivers [2-4] since the isolation of AF sources (by ablation) has been reported to be an effective approach in restoring sinus rhythm $[5,6]$.

Among the proposed techniques, electrocardiographic imaging (ECGI) aims to non-invasively reconstruct the electrophysiological activity on the heart surface from body surface potentials (BSP) [7]. The ECGI is an illposed problem so regularization methods must be applied to obtain stable and realistic solutions [8]. However, the ECGI has not been fully validated for AF driver location. In this work, we propose an assessment procedure based on three performance metrics and apply it to three representative regularization techniques.

The remaining of the paper is organized as follows. In Section 2.1 we present the computational model used for the study, the regularization techniques and the performance metrics. Results are described in Section 3 and Section 4 summarizes the main conclusions.

\section{Methods}

\subsection{Computational models}

We use realistic computational models of atria (2039 nodes) and torso (659 nodes), and simulate different propagation patterns over the atrial surface and the associated BSP $[9,10]$. Two propagation patterns were considered, namely, (i) simple AF propagation pattern (SAF), represented by a right-to-left dominant frequency (DF) gradient with a reentry located in the right atria, at $7.3 \mathrm{~Hz}$ (the rest of the atrial tissue is activated at $4.7 \mathrm{~Hz}$ ); and (ii) complex AF propagation pattern (CAF), with $25 \%$ of atrial cells under fibrotic conditions [4], and a single functional reentry simulated near the RSPV at $6.8 \mathrm{~Hz}$, while the rest of the atria activates at $5.4 \mathrm{~Hz}$. BSP were calculated by using the boundary element method [11], referenced to the Wilson Terminal Center and corrupted with additive Gaussian noise with a signal to noise ratio $S N R=20 \mathrm{~dB}$. 


\subsection{Inverse techniques for driver location}

To estimate the driver location we proceed as follows: first we calculate epicardial potentials by using different regularization approaches. Then, based on the inversecalculated epicardial potential we estimate the phase maps, and from these we compute the location of the driver.

For the first step, we assume the following linear model $\boldsymbol{y}_{t}=\boldsymbol{A} \boldsymbol{x}_{t}+\boldsymbol{\epsilon}$ where $\boldsymbol{\epsilon}$ represents the model residuals. The target is to estimate the epicardial potentials $\mathbf{x}_{\mathbf{t}}$ at time instant $t$, from measurements at the torso $\mathbf{y}_{\mathbf{t}}$ with the knowledge of $\mathbf{A}$. This problem is ill-conditioned and hence a plethora of regularization methods have been proposed to solve it. We next summarize the ones that are considered in this work.

Tikhonov regularization (Tik). For obtaining the epicardial potentials $\boldsymbol{x}_{t}$ at instant $t$, the functional to minimize is:

$$
\left\|\boldsymbol{y}_{t}-\boldsymbol{A} \boldsymbol{x}_{t}\right\|_{2}^{2}+\lambda_{t}\left\|\boldsymbol{x}_{t}\right\|_{2}^{2}
$$

where $\boldsymbol{y}_{t}$ is the vector containing the torso measurements at instant $t$ and $\lambda_{t}$ is the regularization parameter for that time instant, which is computed a priori by using the Lcurve method. The solution of this problem is:

$$
\hat{\boldsymbol{x}}_{t}=\left(\boldsymbol{A}^{T} \boldsymbol{A}+\lambda_{t} \mathbb{I}\right)^{-1} \boldsymbol{A}^{T} \boldsymbol{y}_{t}
$$

Bayesian maximum a posteriori estimation (Bayes). In this method, the spatial covariance matrix of the epicardial potentials can be included as a priori information by making use of the Bayesian MAP estimator [12]. Assuming zero mean for the epicardial potentials, the solution is:

$$
\hat{\boldsymbol{x}}_{t}=\left(\mathbf{C}_{x} \boldsymbol{A}^{T}\right)\left(\boldsymbol{A} \boldsymbol{C}_{x} \boldsymbol{A}^{T}+\boldsymbol{C}_{n}\right)^{-1} \boldsymbol{y}_{t}
$$

where $\boldsymbol{C}_{n}$ is the covariance matrix (diagonal) of the noise, which is assumed i.i.d with constant variance, and $\boldsymbol{C}_{x}$ is the covariance matrix of the epicardial potentials, which is estimated from epicardial potentials taken from a different temporal window than that used for the test.

Greensite (GS). Temporal correlation can also be included in the problem by using the isotropy assumption [13]. Then, the spatio-temporal covariance matrix can be computed as $C_{X}=C_{t} \otimes C_{x}$, where $C_{x}$ is the spatial covariance matrix and $C_{t}$ is the temporal covariance matrix. This covariance matrix is large, so a whitening filter can be applied to the data, and the problem is then solved instant by instant with the MAP approach $[14,15]$.

The second step of the procedure consists on computing the phase maps by using a Hilbert transform applied to the signal in each node. Signals have been previously filtered with a band-pass filter around the dominant frequency (passband from $3 \mathrm{~Hz}$ to $\mathrm{DF}+2 \mathrm{~Hz}$ ), which was computed as in [3]. Finally, the phase maps are used to detect the core of reentrant activity $[3,6]$. A driver is defined

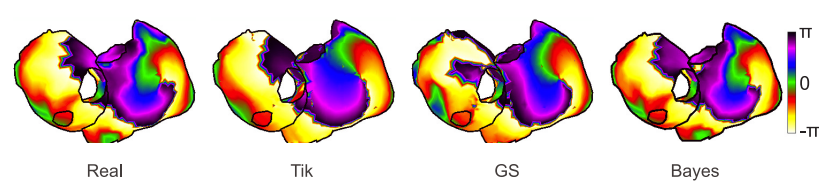

(a)SAF propagation pattern

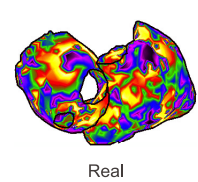

Rea
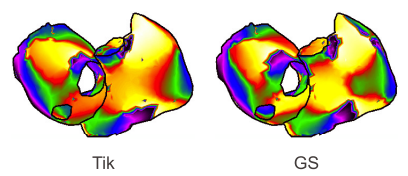

GS

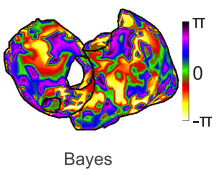

(b)CAF propagation pattern

Figure 1. Real and estimated phase maps with Tik, GS and Bayes, (a) SAF and (b) CAF models.

as the point in a phase map that was surrounded by phases from 0 to $2 \pi$ present at least two full rotations [4]. Finally, dominant driver is defined as the one located in the highest dominant frequency area.

\subsection{Performance metrics}

To compute the performance metrics, the estimated driver location was compared with a gold standard, which was the location of the driver computed from the real epicardial potentials. We propose to estimate the probability of the driver being at each location during an observation time window [16]. First, we estimated the driver location for each instant and built a spatial histogram that represented the number of times the driver was observed at each node. This histogram was normalized to obtain a spatial mass function (SMF) of the driver location, $p(n)$, with $n \in\{1, \ldots, N\}$ the node index. Second, defining the driver region (DR) as the region where the SMF is nonzero, we compared the real and estimated SMFs $(p(n)$ and $\hat{p}(n))$ using three metrics:

- The weighted under-estimation indicator (WUI), defined as the weighted percentage of the true DR that is not detected out of the entire true DR. Our estimate is probabilistic, so we weight the area $A_{n}$ associated with the $n$-th node with the probability of locating the driver in that node, i.e. with $p(n)$. The area associated with one node is the area of the faces surrounding the node. Hence, the WUI indicator is computed as:

$$
W U I(\%)=100 \frac{\sum_{n \in F N} p(n) A_{n}}{\sum_{n \in F N} p(n) A_{n}+\sum_{n \in T P} p(n) A_{n}}
$$

where $F N$ (False Negative) is the set of nodes belonging to the true DR but not to the estimated DR, TP (True Positive) is the set of nodes in both the true and estimated DRs. - The weighted over-estimation indicator (WOI), defined as the percentage of the misjudged DR out of the estimated 
Table 1. Driver location performance for SAF and CAF models for SNR=20: WUI, WOI and $C C_{S M F}$.

\begin{tabular}{lccccccc} 
& \multicolumn{3}{c}{ SAF } & & \multicolumn{3}{c}{ CAF } \\
\cline { 2 - 4 } \cline { 6 - 8 } Tik & WUI & WOI & $C C_{S M F}$ & & WUI & WOI & $C C_{S M F}$ \\
Bayes & 46.33 & 22.48 & 0.52 & & 6.65 & 66.51 & 0.24 \\
GS & 20.68 & 6.53 & 0.78 & & 2.64 & 9.29 & 0.99 \\
& 48.12 & 20.94 & 0.47 & & 6.75 & 65.22 & 0.46
\end{tabular}
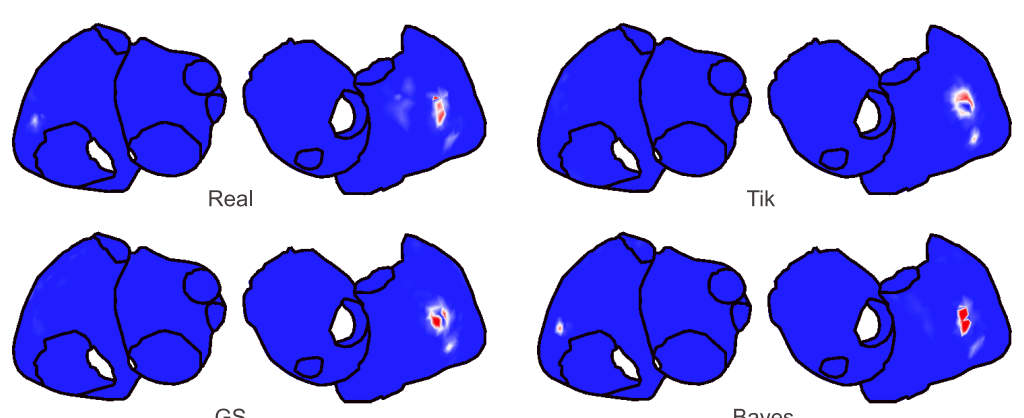

Bayes

(a)SAF propagation pattern
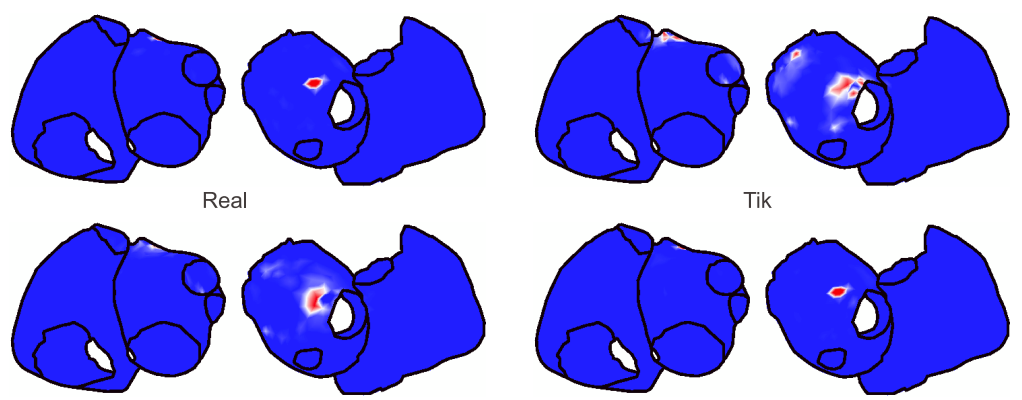

GS

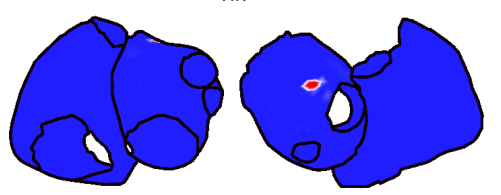

Bayes

(b)CAF propagation pattern

Figure 2. Real and estimated SP location with Tik, GS and Bayes, for (a) SAF and (b) CAF models.

DR, with the same weightings described for the WUI:

$$
W O I(\%)=100 \frac{\sum_{n \in F P} \hat{p}(n) A_{n}}{\sum_{n \in F P} \hat{p}(n) A_{n}+\sum_{n \in T P} \hat{p}(n) A_{n}}
$$

where $F P$ (False Positive) is the set of nodes belonging to the estimated DR but not to the true DR.

- The correlation coefficient between SMFs $\left(C C_{S M F}\right)$ that summarizes location accuracy in one parameter.

The first two metrics are weighted versions of those presented in [17], which are used for ischemia region detection.

\section{Results}

Figure 1 shows the phase maps. In all the cases the Bayes method clearly outperformed the others. Recall that this method used a priori information taken from real epicardial potentials. In all the cases a singularity point was observed where the re-entrant activity was placed.
Figure 2 shows the real and estimated SMF of the driver location. For the SAF model the driver was placed in the right atrium, and moved over a wide region (white areas in Fig 2(a)). All methods were are able to locate the driver most of the time (red areas). For the CAF model the driver locations estimated with Tikhonov and GS methods were more spread out than in the Bayes case, however with very high probability at the right place.

Table 1 shows the WUI, WOI and $C C_{S M F}$ metrics. Bayes and Tik methods performed well in terms of the three metrics for the SAF model (WUI between $20 \%$ and $47 \%$, WOI between $6.53 \%$ and $34 \%, C C_{S M F}$ between 0.52 and 0.78 ). The results varied significantly for the CAF model. While Bayes method still provided good results, Tik method overestimated the DR. Note that the true DR was small in the CAF case, so moderate errors in the location led to high variations in the error metrics. Finally, $C C_{S M F}$ summarized well location accuracy and tended to zero for high values of WUI and/or WOI. 


\section{Discussion and conclusions}

The SMF of the driver location aggregates the results of the location procedure in a time-scale of a few seconds, rather than providing a useless instantaneous measurement of the location accuracy. It is also a probabilistic representation of the location of the driver, so it would be easy to interpret and rather useful in a clinical environment.

The WOI and WUI assess the accuracy of the estimated SMF. They take into account the irregularities of the atria surface by weighting the presence of a driver at one location with the area surrounding the specific node. Also, it takes into account that the driver is not static, and that it is observed in each place a variable number of instants. By comparing the numerical results with the SMF plots, we observed that the WOI and WUI consistently evaluated the similarities between real and estimated SMFs. Finally, the $C C_{S M F}$ summarized the WOI and WUI, since it took a low value when the WOI or the WUI are not high.

Regarding the compared techniques, the Bayes method clearly outperformed the others, due to the use of a priori information. The GS method did not improve the Tik results. Finally, although not shown here, the estimation of the epicardial potentials for the three methods were less reliable than the estimation of the phase maps and the driver location. Since driver location is more useful in the clinical practice than raw epicardial potentials, this is a promising result supporting those obtained in $[2,10]$.

\section{Acknowledgements}

This work has been partially supported by TEC201346067-R (Ministerio de Economía y Competitividad, Spanish Government).

\section{References}

[1] V. Fuster, et al. Acc/aha/esc 2006 guidelines for the management of patients with atrial fibrillation: full text. Europace 2006;8(9):651-745.

[2] Haissaguerre M, Hocini M, Shah AJ, Derval N, Sacher F, Jais P, Dubois R. Noninvasive panoramic mapping of human atrial fibrillation mechanisms: A feasibility report. Journal of Cardiovascular Electrophysiology 2013; 24(6):711-717.

[3] Rodrigo M, Guillem MS, Climent AM, Pedrón-Torrecilla J, Liberos A, Millet J, et al. Body surface localization of left and right atrial high-frequency rotors in atrial fibrillation patients: A clinical-computational study. Heart Rhythm 2014;11(9):1584 - 1591.

[4] Rodrigo M, Climent AM, Liberos A, Calvo D, FernándezAvilés F, Berenfeld O, et al. Identification of dominant excitation patterns and sources of atrial fibrillation by causality analysis. Annals of Biomedical Engineering 2016; 44(8):2364-2376.
[5] Shah AJ, Hocini M, Pascale P, Roten L, Komatsu Y, Daly M, et al. Body surface electrocardiographic mapping for non-invasive identification of arrhythmic sources. Arrhythmia Electrophysiology Review 2013;2(1):16 - 22.

[6] Guillem M, Climent A, Rodrigo M, Fernández-Avilés F, Atienza F, Berenfeld O. Presence and stability of rotors in atrial fibrillation: evidence and therapeutic implications. Cardiovascular Res 2016;109(4):480-492.

[7] Brooks DH, Macleod R. Electrical imaging of the heart. IEEE Signal Process Mag 1997;14:24-42.

[8] Oster HS, Rudy Y. The use of temporal information in the regularization of the inverse problem in electrocardiography. IEEE Trans Biomed Eng 1992;39:65-75.

[9] García-Molla V, Liberos A, Vidal A, Guillem M, Millet J, González A, et al. Adaptive step \{ODE\} algorithms for the $3 \mathrm{~d}$ simulation of electric heart activity with graphics processing units. Computers in Biology and Medicine 2014; 44:15 - 26.

[10] Pedrón-Torrecilla J, Rodrigo M, Climent A, Liberos A, Pérez-David E, Bermejo J, et al. Noninvasive estimation of epicardial dominant high-frequency regions during atrial fibrillation. J Cardiovascular Electrophysiol 2016; 27(4):435-442.

[11] Pedrón-Torrecilla J, Rodrigo M, Climent AM, Liberos A, Pérez-David E, Bermejo J, Arenal Á, Millet J, FernándezAvilés F, Berenfeld O, Atienza F, Guillem MS. Noninvasive estimation of epicardial dominant high-frequency regions during atrial fibrillation. Journal of Cardiovascular Electrophysiology 2016;27(4):435-442.

[12] van Oosterom A. The use of the spatial covariance in computing pericardial potentials. IEEE Transactions on Biomedical Engineering 1999;46(7):778-787.

[13] Greensite F. The temporal prior in bioelectromagnetic source imaging problems. IEEE Transactions on Biomedical Engineering 2003;50(10):1152-1159.

[14] Onal M, Serinagaoglu Y. Spatio-temporal solutions in inverse electrocardiography. In 4th ECFIMBE 23-27 November 2008 Antwerp, Belgium. 2009; 180-183.

[15] Onal M, Serinagaoglu Y. Spatio-temporal solutions in inverse electrocardiography. In 4th ECFIMBE 2008 23-27 November 2008 Antwerp, Belgium. 2009; 180-183.

[16] Haissaguerre M, Hocini M, Denis A, Shah AJ, Komatsu Y, Yamashita S, et al. Driver domains in persistent atrial fibrillation. Circulation 2014;130(7):530-538.

[17] Wang D, Kirby RM, MacLeod RS, Johnson CR. Inverse electrocardiographic source localization of ischemia: An optimization framework and finite element solution. Journal of Computational Physics 2013;250:403 - 424.

Address for correspondence:

Carlos Figuera (carlos.figuera@ urjc.es). Dept. III-D206, Camino del Molino s/n. 28943 - Fuenlabrada (Madrid), Spain. 